\title{
EDITORIAL \\ Geriatric neurosurgery: the unfolding of a new subspecialty
}

\author{
Eric Schmidt, MD, PhD, ${ }^{1}$ Laurent Balardy, MD, ${ }^{2}$ Thomas Geeraerts, MD, PhD, ${ }^{3}$ \\ Nadège Costa, PhD, ${ }^{4}$ Christian A. Bowers, MD, ${ }^{5}$ and Mark Hamilton, MD, CM, FRCSC ${ }^{6}$
}

Departments of ${ }^{1}$ Neurosurgery, ${ }^{2}$ Geriatrics, ${ }^{3}$ Anesthesiology and Critical Care, and ${ }^{4}$ Medical Information, University Hospital of Toulouse, France; ${ }^{5}$ Department of Neurosurgery, University of New Mexico, Albuquerque, New Mexico; and ${ }^{6}$ Department of Clinical Neurosciences, Division of Neurosurgery, Calgary, Alberta, Canada

$\mathrm{T}$ HIS is the first time that an issue of Neurosurgical Focus has been dedicated to the subject of cranial surgery in geriatric patients. This occasion provides us with an opportunity to review the elements of geriatric neurosurgical care that together represent the unfolding of what should be considered a new and extremely relevant neurosurgical subspecialty dedicated to the elderly. While specific issues associated with spinal neurosurgical care are not addressed in this issue of Neurosurgical Focus, the principles presented regarding aging, frailty, and physiological and neurological reserve are nevertheless relevant and applicable.

\section{The Global Impact Associated With an Aging Population}

The world population of individuals aged 65 years and older is growing at a very rapid rate. All surviving baby boomers in Western countries will reach that age by 2030 . In addition, the population of those aged 85 years and older is the world's fastest-growing demographic and will represent $2 \%-3 \%$ of the world population by 2030 . Also in 2030, China's population will consist of more than 200 million people older than 65 years and 66 million people older than 80 years. The estimated cost of brain disorders in the US ${ }^{1}$ and in Europe ${ }^{2}$ is approximately $\$ 800$ billion per year, which is more than the combined costs of cancer, cardiovascular disease, and diabetes. These costs are expected to dramatically increase as the elderly portion of the population is expected to double between 2020 and 2050.

Loss of independence in older individuals results in a considerable direct financial burden. Industrialized countries currently face a significant challenge to efficiently finance healthcare systems. Indeed, while general cost containment is a preoccupation in most countries, these strategies have not been sufficient to counter the funda- mental challenges. ${ }^{3}$ Inpatient hospital care accounts for approximately $60 \%$ of the total annual healthcare costs in the elderly, often secondary to the multiple coexistent chronic diseases with associated disabilities. ${ }^{4,5}$ There is an urgent and considerable need to investigate and develop adapted and personalized care strategies for this age group, and to accomplish this, we should avoid efforts aimed at trying to save resources via simple "productive efficiency," which just uses the least costly processes. Rather, we should attempt to achieve "allocative efficiency" by using a combination of approaches to achieve a level of healthcare that balances resource inputs (costs in the form of labor, capital, or equipment) and either intermediate outputs (number of patients treated, waiting time, etc.) or final health outcomes (lives saved, life-years gained, or qualityadjusted life-years) ${ }^{6}$

\section{Neurosurgery in the Light of a Modern Approach to Aging}

While aging is a normal process and not a disease, a distinction should be made between healthy and pathological aging. ${ }^{7}$ Pathological brain aging is a divergence from healthy brain aging that causes altered sensory or cognitive functions with reduced mobility, impeding daily life and increasing the need for supportive care. A modern approach to aging focuses on efforts to gain a better understanding of pathological aging and the mechanisms that account for any loss of autonomy.

The concepts of physiological and neurological reserve and "frailty" originally introduced in gerontology as a precursor state to functional dependency are applicable to neurosurgery. ${ }^{8}$ Fried's model of frailty, based on identifying five physiological criteria (fatigue, loss of appetite, muscle weakness, slow walking pace, and decreased physical activity), provides a useful operational concept to identify and target populations at risk of dependency so 


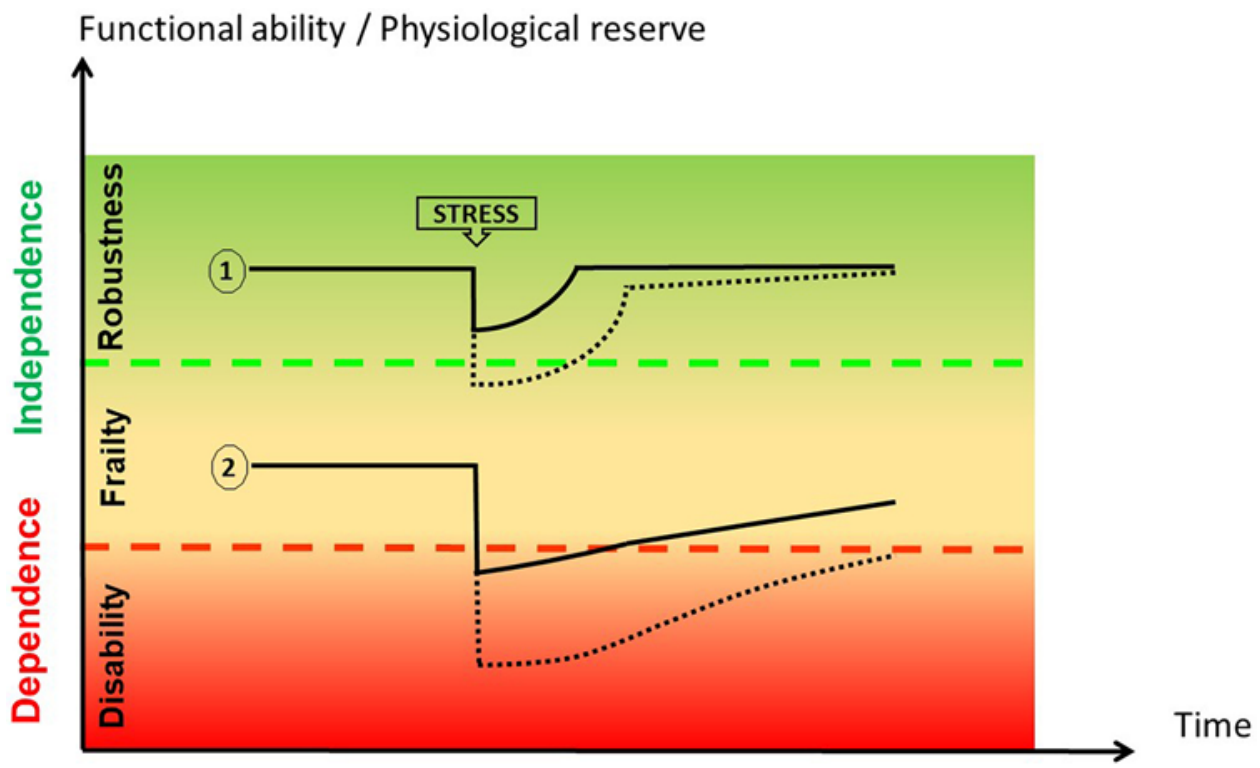

FIG. 1. Vulnerabilities of robust and frail older persons to a minor (solid line) or major (dashed line) change in health status. Based on the work of Calvani et al..$^{10}$ and Clegg et al. ${ }^{11}$

as to anticipate and prevent the processes leading to disablement. ${ }^{9}$ This concept is demonstrated in Fig. 1 (based on the work of Calvani et al. ${ }^{10}$ and Clegg et al. ${ }^{11}$ ), a comparison of the responses of robust and frail persons to a sudden change in health status following minor stress (e.g., a urinary tract infection) or major stress (e.g., a neurosurgical procedure). A robust, independent older person (Fig. 1, no. 1) experiences only a relatively small deterioration in function following minor stress before returning to homeostasis and independence. Faced with greater, or major, stress such as a neurosurgical procedure, this patient's recovery to independence requires more physiological reserve and a longer time to attain the initial prestress condition. The frail patient (Fig. 1, no. 2) may not return to prestress baseline homeostasis following even minor stress. After experiencing greater stress such as a neurosurgical procedure, a frail elderly person with exhausted physiological and neurological reserve quickly becomes disabled, with a significantly lower probability of recovering independence. The probability of long-term recovery to the initial prestress condition is low. These concepts of frailty, functional ability, and physiological and neurological reserve need to be fully appreciated to assist in the avoidance of poor outcomes and to promote quality of life for all populations.

Continuing with this theme, a major objective of the modern approach to aging focuses on mechanisms leading to individual loss of independence. For example, Sacanella et al. looked at the survival of 230 healthy elderly patients who had been admitted to a medical ICU. ${ }^{12}$ They determined that $70 \%$ of these patients were alive at the time of discharge from the hospital, but only $49 \%$ were alive 1 year after discharge. Among the 1-year survivors, there was a twofold increase in the prevalence of geriatric syndromes (e.g., delirium, falls, immobility, pressure ulcers, malnutrition, cognitive impairment, polypharmacy, and urinary or fecal incontinence). Many older patients worsen with regard to independence after discharge from neurosurgical treatment, even though an optimal surgical procedure may have been performed. Current evidence suggests that the older the patient, the poorer the functional outcome. A geriatric patient is more likely to develop new functional deficits during hospitalization. A more holistic and geriatrics-focused care model must be adopted to correct this deficiency.

\section{Geriatric Neurosurgery: An Evolving Framework}

Neurosurgery, as a medical specialty concerned with the prevention, diagnosis, and surgical treatment of brain disorders, as well as rehabilitation, can help alleviate the burden of pathological brain aging and its societal impact. The history of neurosurgical subspecialization is important for context. Pediatric neurosurgery, as the first neurosurgical subspecialty that was created, developed from the need to address the unique requirements of children and the realization that application of neurosurgical rules guiding treatment of adults to children ignored the vast and fundamental differences between the age groups. Much has been gained by embracing rather than ignoring the specificity and, at times, the uniqueness of the pediatric neurosurgical condition and the adoption of a multidisciplinary approach to the neurosurgical care of children. The benefits of working in a pediatric hospital are significant. The focus is on child and family-centered care. The care is multidimensional and there is a shared awareness of the unique elements associated with providing care with a goal to improve outcomes for children. Last, the awareness of the specific medical and surgical needs of children shared by pediatricians, pediatric anesthetists, pediatric ICU personnel, pediatric nurses, and pediatric neurosurgeons is a palpable benefit and a hard-earned achievement. At the other end of the age spectrum, the 
geriatric neurosurgical condition should be recognized for its own specificity and uniqueness, which requires both a multidisciplinary approach and the advanced technical skills possible in a geriatrics-centric environment.

The recognition of geriatric neurosurgery as a subspecialty is currently lagging behind that of pediatric neurosurgery. This can be partially illustrated by an examination of published medical literature. It is, however, important to identify the difference between simple recognition of the problems associated with pathological aging and delineation of a process to address these issues while preventing complications and any loss of independence. Using PubMed and the search criteria "neurosurgery + elderly," we identified 6896 references in 2019 in the MEDLINE database, demonstrating the strong (typically negative) impact of pathological aging in neurosurgery. Using the search criteria "neurosurgery + geriatric," with the term "geriatric" used to indicate a potential process to address issues specific to the elderly, only 129 references were identified. Publications between 1960 and 2020 demonstrate a steadily increasing trend in the number of citations combining "neurosurgery + elderly," contrasting with a flat trend of citations for "neurosurgery + geriatric." As a matter of comparison, performing the same PubMed analysis in 2019 with "neurosurgery + child" and "neurosurgery + pediatric," with "pediatric" used to indicate a potential process to address issues specific to children, there were 1651 references for "neurosurgery + child" and 2045 for "neurosurgery + pediatric." The trend from 1960 until 2020 shows citations for each of these categories nicely converging. This surrogate measurement illustrates both the success and growth that has occurred in pediatric neurosurgery subspecialty care and offers a goal for the future development of geriatric neurosurgery as a subspecialty.

We propose five necessary steps to improve and foster the development of geriatric neurosurgery as a successful subspecialty:

1. Develop accepted common terminologies to improve the quality of relevant research and publications and foster the successful sharing of competence.

2. Develop, evaluate, and widely implement relevant clinical tools to identify and mitigate the issues of frailty and physiological and neurological reserve associated with neurosurgically relevant disorders and procedures.

3. Adapt neurosurgical and anesthetic procedures to mitigate specific issues identified for the geriatric patient.

4. Establish and implement comprehensive pre-, peri-, and postoperative management strategies to target maintenance of global autonomy with the aim to prevent hospitalization-associated disability.

5. Assess the educational needs and requirements necessary for doctors, nurses, and allied healthcare workers to allow successful implementation of these assessment strategies and patient care models, with an additional goal to foster relevant and successful research.

\section{Neurosurgical Care for the Elderly Patient: A General Neurosurgery Versus a Geriatric Neurosurgery Pathway}

It is useful to illustrate the differences an elderly pa- tient might experience when facing management of a neurosurgical disorder following the current and more common general neurosurgery model contrasted with what a geriatric neurosurgical model of care can and should offer (Fig. 2).

\section{Pathway of an Elderly Patient in General Neurosurgery}

The current, typical general neurosurgery care pathway, as opposed to one specific to geriatric neurosurgery, is illustrated in Fig. 2 using numbered steps. After the identification of the need for a neurosurgical procedure (step 1), preoperative anesthetic assessment (step 2) and patient preparation for surgery typically occur according to a standardized protocol. Elderly patients require particular attention during this stage (step 3) due to their combination of age, comorbidities, and frailty. Elderly patients are potentially more vulnerable to stresses of both acute illness ${ }^{13,14}$ and issues associated with the hospitalization process,${ }^{15}$ making them prone to treatment-associated complications. Autonomy, frailty, physiological reserve, and comorbidities should be taken into consideration when assessing the treatment risk-benefit analysis. Anticipation of the duration of the hospital stay linked with a discharge planning strategy, as well as the identification and prevention of age-specific complications, is crucial in the older population. During hospitalization, the elderly patient's case is typically managed by the neurosurgeon (step 4) and anesthetist (step 5). Surgical procedures and anesthetic protocols, if not tailored to the patient's age, physiological condition, and frailty, can increase complications (step 6), resulting in an extended length of hospital stay and loss of independence. During hospitalization, nurses (step 7) provide essential care for the patient and play a vital role in the screening and prevention of specific complications associated with geriatric patients, such as an increased risk for falls, delirium, or incontinence (step 8 ). When care is not geared to the specific needs of the geriatric population, loss of autonomy occurs and is associated with increased delays in hospital discharge (step 9) and increased requirements for home care service or failure to return home with placement in an alternative care facility (extended gray zone of dependence). Finally, if inadequately designed outcome measures are used, the postneurosurgical evaluation of an elderly patient (step 10) can underestimate loss of autonomy. Hence, mid- and long-term outcomes of a skillfully performed surgical procedure can be overshadowed by the patient's loss of independence with poor quality of life (step 11).

\section{Pathway of an Elderly Patient in Geriatric Neurosurgery}

The process and potential benefits of the recommended geriatric neurosurgery (vs general neurosurgery) care pathway are also illustrated in Fig. 2. After identification of the potential need for the neurosurgical procedure by the neurosurgeon (step 12), a collaborative process is undertaken with the anesthetist (step 13) and geriatrician (step 14). This can occur in parallel to ensure that the benefit of the anticipated procedure is neither under- nor overestimated, leading ultimately to a patient-centric collaborative decision to proceed or not to proceed with surgery. A neurosurgical decision regarding an elderly patient should 


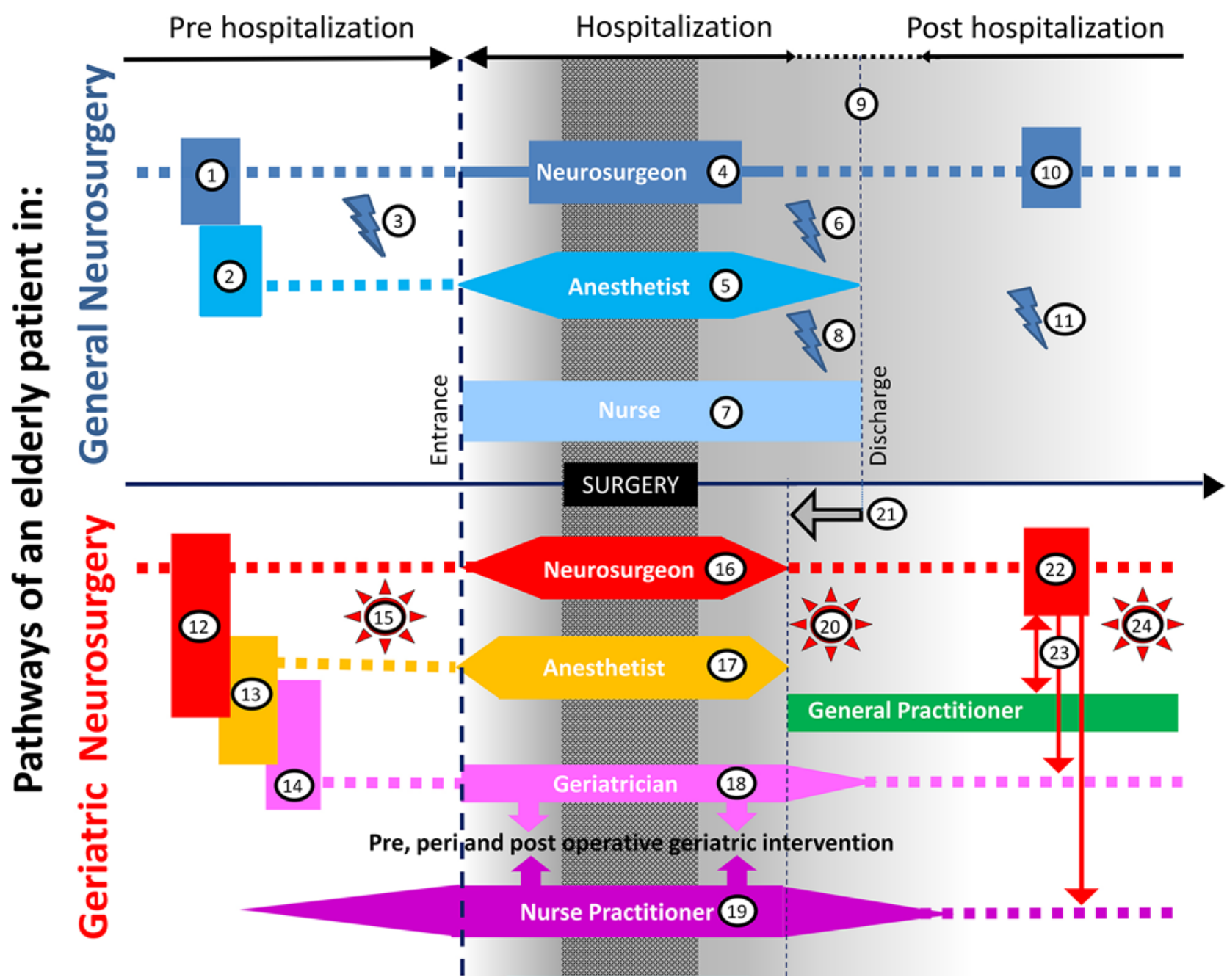

FIG. 2. Pathways of an elderly patient: contrasting a general neurosurgery emphasis with a geriatric neurosurgery one. The numbered steps are explained in the section titled "Neurosurgical Care for the Elderly Patient: A General Neurosurgery Versus a Geriatric Neurosurgery Pathway."

endeavor to find the right answer for each patient. Chronological age is not per se a defining limitation. Particular attention is paid to the concepts of frailty and physiological and neurological reserve and to the risks of all comorbidities using dedicated and validated screening tools. To optimize case management, a geriatrics-relevant evaluation should, when possible, be undertaken prior to hospitalization. Preoperative preparation is undertaken using specific protocols dedicated to the geriatric patient (step $15)$. Once hospitalized, the case is managed by a multidisciplinary team that combines the expertise and skills of neurosurgery within a geriatric culture. The neurosurgeon (step 16) optimizes the surgical procedure to balance the treatment need with the patient's capabilities and realistic disease-specific outcomes. The anesthetist (step 17) may need to modify the anesthetic protocol before, during, and after surgery to avoid, in particular, drug-induced side effects and polypharmacy. Ideally, the geriatrician (step 18) helps manage the patient's in-hospital neurosurgical pathway, and a specialized nurse practitioner (step 19) helps coordinates geriatric care. Postoperative recovery and ward nurses should be trained to use relevant assessment and care tools for the prevention and early identification of geriatric patient issues. The methods should include teaching patients how to retain their independence and self-care during the hospital stay and after discharge. Particular attention is paid to preventing falls, delirium, and incontinence (step 20). Identification of a frail patient with a high likelihood of developing hospital-associated disability should be accomplished as early as possible by using this process. An integrated strategy with planning of anticipated discharge is required to reduce the length of stay and rehospitalization (step 21), including the early organization of home care service or alternative options if a non-home destination is probable (reduced gray zone of dependence). After leaving the hospital, communication with the patient by a nurse practitioner and integration with the patient's general practitioner are essential. Postdischarge neurosurgical evaluation should include the use of dedicated and validated screening tools (step 22), 
engaging the geriatrician or nurse practitioner when necessary (step 23), to include ecological assessments targeting the patient's autonomy, quality of life, and well-being (step 24).

\section{Conclusions}

It is obvious that successful neurosurgery in the geriatric patient involves more than just the performance of a neurosurgical procedure. While this may seem intuitive, in practice this is not always realized. To achieve optimal patient-centric care requires a medicosurgical collaborative multidiscipline endeavor to optimize preoperative evaluation, tailor surgical goals, and improve postoperative care for the short- and long-term benefit of the patient and family. This requires the combined expertise and skills of neurosurgeons and anesthetists, supported by gerontologists and other allied healthcare providers before, during, and after the surgical procedure. Geriatric neurosurgery is unfolding as a new subspecialty to meet the needs and demands of this ever-growing neurosurgical healthcare issue, and the papers in this issue represent an important, but by no means final, step forward in this process.

https://thejns.org/doi/abs/10.3171/2020.7.FOCUS20509

\section{References}

1. Gooch CL, Pracht E, Borenstein AR, et al. The burden of neurological disease in the United States: a summary report and call to action. Ann Neurol. 2017;81(4):479-484.

2. Olesen J, Gustavsson, Svensson M, et al. The economic cost of brain disorders in Europe. Eur J Neurol. 2012;19(1): 155-162.

3. Brown LD. Comparing health systems in four countries: lessons for the United States. Am J Public Health. 2003;93(1): $52-56$.

4. Larsen FB, Pederson MH, Friis K, et al. A latent class analysis of multimorbidity and the relationship to socio-demographic factors and health-related quality of life. A national population-based study of 162,283 Danish adults. PLoS One. 2017;12(1):e0169426.

5. Hazra NC, Rudisill C, Gulliford MC. Determinants of health care costs in the senior elderly: age, comorbidity, impairment, or proximity to death? Eur J Health Econ. 2018;19(6): 831-842.
6. Palmer S, Torgerson D. Economic notes: definitions of efficiency. BMJ. 1999;318(7191):1136.

7. Rowe JW, Kahn RL. Successful Aging. Pantheon Books; 1998.

8. Pazniokas J, Gandhi C, Theriault B, et al. The immense heterogeneity of frailty in neurosurgery: a systematic literature review. Neurosurg Rev. Published online January 17, 2020. doi:10.1007/s10143-020-01241-2

9. Fried LP, Tangen CM, Walston J, et al. Frailty in older adults: evidence for a phenotype. J Gerontol A Biol Sci Med Sci. 2001;56(3):M146-M156.

10. Calvani R, Marini F, Cesari M, et al. Biomarkers for physical frailty and sarcopenia: state of the science and future developments. J Cachexia Sarcopenia Muscle. 2015;6(4):278-286.

11. Clegg A, Young J, Iliffe $S$, et al. Frailty in elderly people. Lancet. 2013;381(9868):752-762.

12. Sacanella E, Pérez-Castejón JM, Nicolás JM, et al. Functional status and quality of life 12 months after discharge from a medical ICU in healthy elderly patients: a prospective observational study. Crit Care. 2011;15(2):R105.

13. Caplan GA, Williams AJ, Daly B, Abraham K. A randomized, controlled trial of comprehensive geriatric assessment and multidisciplinary intervention after discharge of elderly from the emergency department-the DEED II study. $J \mathrm{Am}$ Geriatr Soc. 2004;52(9):1417-1423.

14. Ellis G, Gardner M, Tsiachristas A, et al. Comprehensive geriatric assessment for older adults admitted to hospital. Cochrane Database Syst Rev. 2017;9(9):CD006211.

15. Matthews SB, Arnold SE, Epperson CN. Hospitalization and cognitive decline: can the nature of the relationship be deciphered? Am J Geriatr Psychiatry. 2014;22(5):465-480.

\section{Disclosures}

The authors report no conflict of interest.

\section{Correspondence}

Eric Schmidt: schmidt.e@chu-toulouse.fr.

INCLUDE WHEN CITING

DOI: 10.3171/2020.7.FOCUS20509. 